\title{
$\mathrm{N} \mathrm{ACO}=$ \\ Anteratan

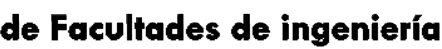

http://www.educacioneningenieria.org

\section{La lectura y la escritura como herramientas pedagógicas para la enseñanza de Ingeniería de Software}

\author{
Royer David Estrada-Esponda \\ Grupo de Investigación Leer, Escribir y Pensar, Universidad del Valle, Tuluá, Colombia. royer.estrada@correounivalle.edu.co
}

\begin{abstract}
Resumen - El presente artículo expone los resultados de la intervención del curso de Desarrollo de Software II en la Universidad del Valle sede Tuluá, que consistió en la incorporación de instrumentos pedagógicos relacionados con la lectura y la escritura, tales como lectura modelada, elaboración de resúmenes, revisión entre pares y exposiciones orales. Esto con el fin de facilitar la adquisición y creación de conocimientos alrededor de la Ingeniería de Software, con la consigna de generar espacios con enfoques constructivistas y orientados al aprendizaje colaborativo. Lo anterior en el marco de un enfoque metodológico de investigación-acción, que posibilitó la interacción entre el grupo de estudiantes del curso objeto de intervención y el profesor a cargo. En consecuencia, se generó una realimentación continua en pro del mejoramiento en la calidad de los procesos de enseñanza y aprendizaje y, por tanto, en la calidad académica.
\end{abstract}

Palabras Clave- Lectura, Escritura, Ingeniería de Software, Pedagogía.

Recibido: 31 de mayo de 2017. Revisado: 16 de junio de 2017. Aceptado: 29 de junio de 2017.

\section{Reading and writing as pedagogical tools for teaching Software Engineering}

Abstract- This article presents the results of the intervention of the course "Software Development II" at Tuluá University, which included the incorporation of pedagogical tools related to reading and writing, such as modeling reading, abstracts, Peer review and oral exposures. It is to facilitate the acquisition and creation of knowledge around Software Engineering, with the slogan of generating spaces with constructivist approaches and oriented to collaborative learning. The above mentioned, within the framework of a methodological approach of research-action, which made possible the interaction between the group of students of the course of intervention and the teacher in charge. Consequently, a continuous feedback was generated in favor of the improvement in the quality of the teaching and learning processes and, therefore, in the academic quality.

Keywords: Reading, writing, pedagogy, Software Engineering.

\section{Introducción}

De manera equivocada se ha creído que los procesos de lectura y escritura son estáticos, es decir, que reposan en los conocimientos, habilidades y competencias adquiridas en tempranos momentos de la vida, específicamente mientras los individuos transitan por la educación básica y permanecen en su núcleo familiar. Por el contrario, se encuentra en [1] que los diversos contextos en los que se lee y se escribe plantean desafíos y exigen continuar aprendiendo a leer y a escribir durante todas las etapas de formación académica, tales como básica primaria, secundaria, pregrado e incluso en estudios de posgrado.

Por tanto, resulta significativo comprender que los procesos de escritura y lectura son dinámicos y por ello están en constante evolución, lo cual implica que los individuos que acogen dichos procesos deben permanecer en continuo aprendizaje y refinamiento de sus prácticas de escritura y lectura.

Paralelamente, no solo la falta de conciencia sobre la naturaleza en sí de los procesos de lectura y escritura es la única responsable de la problemática actual en la educación superior, representada en las debilidades de dichos componentes en los estudiantes que transitan por ella, de hecho la poca cultura académica de los estudiantes no contribuye con la formalización de habilidades de esa dimensión. Lo anterior resulta significativo en la educación superior pública, debido a que la mayoría de sus estudiantes pertenecen a estratos socioeconómicos bajos, lo cual conduce a considerar que si los estudiantes provienen de familias de escasos recursos económicos, o sus padres tienen un bajo o nulo capital cultural en su estado objetivado, es explicable que dicha población no manifieste prácticas escolares que garanticen un desempeño y tránsito idóneos por los estudios profesionales [2]. Para el caso específico de la sede Tuluá de la Universidad del Valle, el 86\% de sus estudiantes pertenecen a estratos socioeconómicos nivel 1 y 2, según información encontrada en [3], lo cual, sin lugar a duda, invita a considerar dicha realidad en procesos de intervención como los presentados en este artículo.

Por otra parte, y específicamente en el campo de la Ingeniería de Software, es necesario proveer desde las universidades herramientas que fortalezcan las prácticas discursivas de los estudiantes, de modo que ellos, una vez se introduzcan en un entorno laboral, puedan desenvolverse de manera adecuada desde la oralidad propia de su disciplina. También y según lo mencionado en [4], el desempeño del ingeniero en el ámbito profesional demanda, además de saberes

Como citar este artículo: Estrada-Esponda, R.D., La lectura y la escritura como herramientas pedagógicas para la enseñanza de Ingeniería de Software. Educación en Ingeniería, 12(24), pp. 83-88, Julio, 2017. 
técnicos específicos, saberes del dominio del lenguaje para poder transmitir y gestionar la información con la que realizará su trabajo. Así mismo, en [5] se encuentra que en el desarrollo de software es posible identificar dos tipos de conocimiento, el primero orientado a las capacidades creativas y el segundo relacionado con el conocimiento de productos y procesos asociados al dominio del problema.

Sumado a lo anterior, el crecimiento vertiginoso de las Tecnologías de la Información y Comunicaciones (TICs) está generando nuevas formas de trabajo y modificando diversas prácticas en la vida cotidiana de las personas. En esta transformación tecnológica se observa una tendencia progresiva hacia la colaboración entre personas para alcanzar un objetivo común, para lo cual el trabajo se organiza en equipos y cada integrante interactúa con el resto del grupo para obtener una mejor productividad [6]; lo anterior conduce a reflexionar sobre la importancia de las habilidades de escritura y lectura a las que están llamados los estudiantes de la educación superior para hacer que sus procesos académicos y laborales sean más efectivos.

El objetivo de este artículo es presentar una experiencia sobre la incorporación de prácticas referentes a la escritura y la lectura en la asignatura Desarrollo de Software II, que configura un proceso de innovación académica y pedagógica. El documento está estructurado de la siguiente manera: primero se presenta el curso que fue intervenido, luego se comenta una iniciativa desarrollada en la sede Tuluá en el marco de un programa de permanencia, seguido a ello se menciona la metodología utilizada para la intervención del curso Desarrollo de Software II, después se presentan las prácticas e instrumentos utilizados, $\mathrm{y}$, finalmente, se exponen los resultados y conclusiones de la intervención realizada.

\subsection{Descripción del curso}

El curso Desarrollo de Software II es ofrecido en los programas académicos de Tecnología en Sistemas de Información e Ingeniería de Sistemas. La Tabla 1 presenta los datos más relevantes del mismo.

El curso es programado en el quinto semestre del programa tecnológico y en el séptimo semestre del programa profesional, esto advierte sobre el tipo de población que asiste como audiencia a dicho curso, es decir, estudiantes de semestres

Tabla 1

Información del curso

\begin{tabular}{lc}
\hline Código & 750092M \\
\hline Créditos & 4 \\
Intensidad Horaria & 4 horas semanales \\
Habilitable & No \\
Validable & Sí \\
Prerrequisitos & Desarrollo de Software I - \\
& Base de datos \\
Tipo de Asignatura & Área Profesional - \\
& Obligatoria déptimo de Ingeniería / \\
Semestre(s) & Quinto de Tecnología \\
& Ingeniería de Sistemas y \\
Ofrecido a: & Tecnología en Sistemas de \\
& información \\
\hline
\end{tabular}

Fuente: Del autor superiores que para el primer caso se acercan a su momento de graduación y el segundo, estudiantes que se aproximan al inicio de su trabajo de grado.

A propósito de la intervención que se realizó, es pertinente mencionar que en los programas en los que se ofrece el curso se identificaron problemáticas tales como:

Para el caso del programa tecnológico, la problemática reside en que los estudiantes, próximos a la culminación de su proceso formativo, siguen manifestando problemas inherentes con la interpretación y producción de textos académicos, incluso los referentes a su área disciplinaria.

En lo que concierne a estudiantes del programa profesional, se observa que la mayor problemática se ve materializada cuando inician su proceso de trabajo de grado; este proceso se inicia un semestre después de haber finalizado y aprobado el curso objeto de intervención. La problemática en este caso está relacionada con la mencionada en el párrafo anterior, es decir, problemas con la interpretación y producción de textos académicos, pero esta vez enfocados con los temas de interés seleccionados para el trabajo de grado.

También es importante resaltar cómo los objetivos, tanto el general, como algunos específicos del curso están significativamente relacionados con la escritura y la lectura. A continuación se presentan algunos de dichos objetivos.

\subsubsection{Objetivo general}

Capacitar al estudiante para definir, evaluar y mejorar procesos de construcción de software, aplicando estándares de calidad definidos.

\subsubsection{Objetivos específicos}

Escribir el alcance, las responsabilidades, la secuencia e interacción de los procesos para el Sistema de Gestión de Calidad de un grupo desarrollador de software.

Escribir, para el proyecto de software, la especificación de requerimientos, su plan de desarrollo y el contrato.

Comparar y evaluar lo ejecutado contra el plan previsto para el desarrollo, y formular propuestas de mejoramiento para los procedimientos.

Los objetivos anteriormente relacionados dan cuenta de la necesidad que tienen los educandos de desarrollar habilidades de lectura y escritura con el fin, en primera medida, de poder escribir documentación, requerimientos, responsabilidades e informes técnicos asociados a su área disciplinaria, y ser capaces de comprender su contexto con la intención de poder formular alternativas de mejoramiento para sus interesados o clientes. El periodo en el cual se realizó la intervención fue el de febrero-junio 2017 con 21 estudiantes matriculados.

\subsection{Antecedentes}

Antes de dar cuenta del proceso llevado a cabo durante el curso de Desarrollo de Software II, resulta conveniente destacar cómo la Universidad del Valle sede Tuluá ha implementado en el marco de un programa de permanencia y éxito académico, aulas de lectura y escritura con el fin de abordar las problemáticas inherentes a tales procesos. De igual forma, es 
preciso destacar que dichas aulas son ofrecidas como sesiones adicionales a los cursos de lenguaje, español o afines; por tanto, es claro que solo estudiantes de semestres inferiores en los que se ofrecen esos cursos, según las mallas curriculares, representan la población objetivo del mencionado programa, lo cual implica que estudiantes de semestres avanzados no tienen espacios de esa naturaleza para fortalecer habilidades al tenor de la escritura y la lectura, específicamente en sus áreas disciplinarias. Además, las aulas propuestas por la universidad son extra clase, lo cual provoca que las actividades que se desarrollan allí no necesariamente estén ubicadas en el contexto específico del programa académico de los estudiantes.

Por lo anterior y teniendo en cuenta que en cada disciplina se desarrolla una cultura discursiva a la luz de sus particularidades, es necesario mencionar que no es suficiente con que la labor de mejorar las habilidades de lectura y escritura sean atendidas por las universidades por medio de programas como el mencionado anteriormente; como bien se encuentra en [7] es necesario que todos los profesores se comprometan con introducir a sus estudiantes en una oralidad apropiada a cada disciplina, con el fin de generar en ellos elementos de valor que contribuyan a la construcción de una verdadera cultura discursiva.

Paralelamente es necesario hacer énfasis en la necesidad que tienen las instituciones educativas por adoptar prácticas que no solo instrumentalicen la escritura y la lectura en las aulas, incluso en [8] se indica que el problema de la escritura no es de los universitarios, escritores experimentados o incluso estudiantes en fases de pre-universidad y posgrado, sino de la enseñanza en sí misma, del modelo educativo en el que vivimos, de las instituciones que "han tendido a usar la escritura sólo en forma instrumental”. Así pues, es indispensable reflexionar sobre el rol docente en la actualidad, de modo que desde dicho ejercicio pueda ser posible establecer mecanismos o dispositivos pedagógicos que permitan hacer de la escritura un elemento no solo meramente instrumental, por el contrario, entender que dicho proceso tiene alcances hasta lo epistémico, es decir, la escritura como generadora de conocimiento.

Finalmente, y teniendo en cuenta que la Universidad por medio de programas de permanencia o éxito académico comprende la problemática que permea la educación superior en relación con la lectura y la escritura, es menester de los profesores asimilar prácticas relacionadas con tales procesos y estudiar o analizar constantemente cómo dichas prácticas influyen en los estudiantes y en su propio desempeño docente. En esta dirección, la Universidad del Valle, específicamente la Dirección de Autoevaluación y Calidad Académica (DACA), ofreció en la sede Tuluá durante el periodo agosto-diciembre del 2016 el diplomado denominado "La lectura y la escritura en el aula universitaria”, allí un grupo docente de la sede tuvo la oportunidad de discutir el alcance de la lectura y la escritura como elementos potenciadores de la enseñanza y aprendizaje y posibilitó a dicho grupo la construcción o adaptación de los enfoques académicos a la luz de tales elementos. Sin lugar a dudas, lo comentado anteriormente es un insumo para la puesta en marcha de iniciativas como la expuesta en el artículo.

\section{Metodología}

La metodología utilizada para la materialización de la intervención que aquí se presenta corresponde a un enfoque de investigación-acción, con el que se buscó emitir la interpretación de la realidad del curso Desarrollo de Software II, en relación con la lectura y la escritura, desde la mirada no sólo del profesor sino también desde los estudiantes. De hecho, en [9] se enfatiza que la investigación-acción interpreta lo que ocurre desde el punto de vista de los participantes de la investigación; lo anterior significó un valor agregado al ejercicio investigativo, ya que al conocer la perspectiva de los estudiantes se contribuyó también con el mejoramiento continuo del profesor, lo que implica un aumento en la calidad académica que a su vez impactará positivamente en los estudiantes. La investigación acción se constituye en una excelente herramienta para mejorar la calidad académica en la educación. [10]

De igual modo, uno de los propósitos de la investigaciónacción en el contexto de la intervención realizada fue profundizar la compresión del profesor sobre los problemas que surgen en el aula, así pues, dicho enfoque metodológico adoptó un alcance exploratorio, este último resultó significativo para facilitar la realimentación continua con los estudiantes que participaron de la investigación, por tanto, dentro del proceso metodológico fue incluido un sondeo con el fin de conocer la percepción de los estudiantes acerca de las actividades de escritura y lectura desarrolladas durante el curso.

También y paralelamente con las actividades del curso se definieron las siguientes actividades con el fin de incorporar la lectura y la escritura en la cotidianidad de las sesiones:

Inicialmente se asignó la elaboración y presentación de una exposición oral ante todo el grupo de estudiantes como auditorio, para ello se les suministró a todos los grupos conformados una rejilla de evaluación que usaron como insumo para la preparación de su exposición, dicha rejilla entre otros aspectos indicaba cómo debía ser el inicio y cierre de la presentación, cómo sería valorado el uso excesivo de muletillas, el contacto visual con el auditorio, el lenguaje corporal, la seguridad, el tono de voz, la velocidad y el conocimiento del tema.

Adicionalmente, se realizaron tres lecturas modeladas asociadas al curso. En esta actividad fue posible comprender las ideas de los autores, identificar contextos y familiarizar a los estudiantes con contribuciones prácticas y teóricas de su disciplina, de igual modo se propició la participación de los estudiantes durante cada sesión, por medio de la lectura de fragmentos del texto o la intervención con base en sus interpretaciones. Así pues, surgieron preguntas que fueron respondidas por el docente e incluso de alguna u otra manera por los mismos estudiantes, en consecuencia se generó un espacio constructivista en el marco del trabajo colaborativo.

Una vez las lecturas fueron desarrolladas, se asignó la elaboración de resúmenes, con el propósito de verificar la capacidad de síntesis y por tanto detectar si las lecturas modeladas aportaron a la apropiación de ideas y conceptos inmersos en los textos revisados con anterioridad. Para ello se proporcionó una rejilla de evaluación con el fin de esclarecer las características de un resumen y su forma de calificación. Entre los aspectos más importantes de dicha rejilla se destaca que para los estudiantes era explicito cómo iban a ser evaluados y cómo está compuesto un texto tipo resumen, por tanto los resúmenes fueron evaluados en cuanto a contenido y aspectos 
lingüísticos. Vale la pena destacar que las lecturas que fueron utilizadas para el ejercicio, corresponden a artículos de revistas indexadas, no mayores a 12 páginas.

Por otra parte, a medida que los resúmenes fueron elaborados se procedió a establecer un tiempo de la sesión para la co-evaluación de dichos textos, el tiempo promedio para tal actividad fue de 20 minutos. En consecuencia los estudiantes con la ayuda de la rejilla pudieron realizar la evaluación y por tanto se logró concientizar a los mismos sobre la importancia de reconocer al otro como sujeto de aprendizaje en una dinámica constructivista.

También y con el fin de exponer la importancia de la escritura, se proporcionó una plantilla para la elaboración de fichas resúmenes, en ellas los estudiantes podían consignar las ideas principales de las clases con el fin de tener dicho material para las evaluaciones del curso. En cada clase los estudiantes podían entregar una ficha resumen, de ninguna manera se recibieron fichas por fuera de la clase, esto posibilito que la audiencia del profesor tuviera una motivación adicional por tomar nota o incluso apropiar los conceptos desarrollados durante cada sesión. En consecuencia en el evento de evaluaciones los estudiantes podían solicitar las fichas que previamente habían elaborado.

Finalmente y con el propósito de poner en contexto la escritura, se les asignó a los estudiantes en el marco de su proyecto de curso, entregar documentación asociada a un producto software que debían desarrollar, esto teniendo en cuenta que el software está compuesto por estructuras de datos, soporte lógico y documentación. Así pues, de manera explícita, se solicitaron 3 documentos, un manual de usuario, un manual de programador y un procedimiento para el control de versiones, todos los documentos fueron co-evaluados por otros estudiantes, esto con el fin de comprender la importancia de la escritura incluso en su futura labor profesional. Como criterio de evaluación se definió el nivel de comprensión de los documentos, así pues, se dejó claro que la escritura no es automática, y por tanto es resultado de un continuo proceso de mejoramiento.

A continuación, la Fig. 1 presenta el resumen de las actividades de lectura y escritura incluidas en el curso.

Adicionalmente, y entendiendo la necesidad de reconocimiento que habita en los individuos, se usó la página https://www.canva.com/ para la generación de poster o tarjetas de felicitaciones de parte del profesor que fueron publicadas en el campus virtual de la Universidad. Lo anterior entendiendo que en el mundo académico no solo impera la lógica, la razón y el intelectualismo frio, en ese mundo, y sobre todo cuando las actividades académicas dependen significativamente de la personas, los afectos y la cognición son determinantes para el desarrollo de dichas actividades [11].

\section{Resultados y discusión}

Con el fin de presentar los resultados y en consecuencia generar una discusión, es relevante exponer que fue necesaria la aplicación de un instrumento para la determinación de la percepción de los 21 estudiantes en relación con las actividades desarrolladas durante el curso. A continuación se presentan las características principales de dicho instrumento.

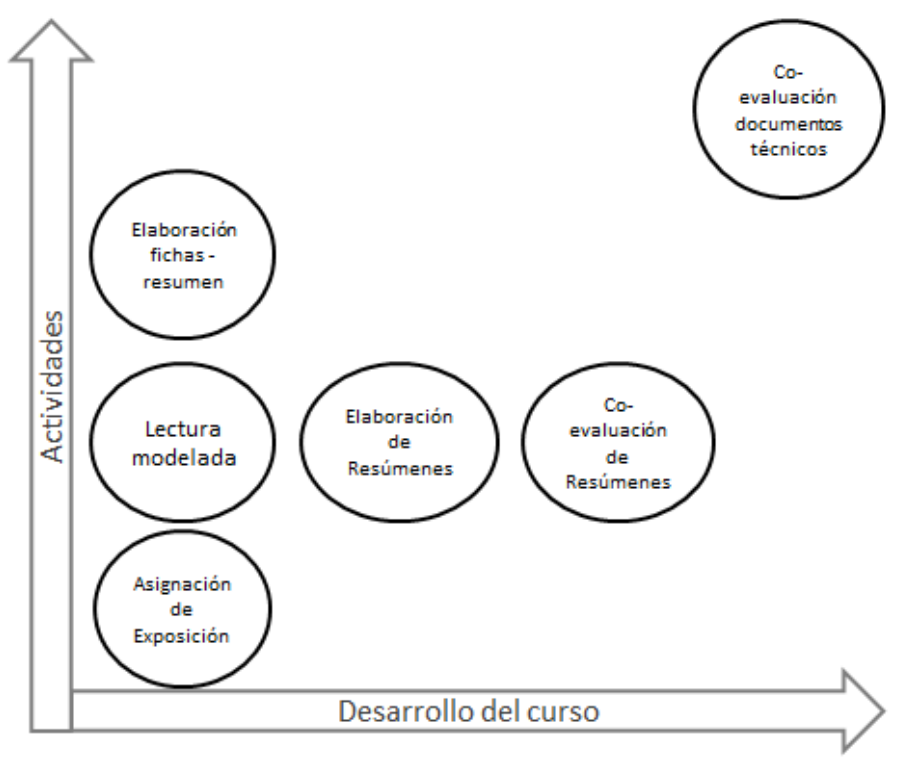

Figura 1. Actividades incluidas en el curso.

Fuente: Del autor

En primera medida fue necesario definir un objetivo para cada una de las preguntas que se consultarían a los estudiantes, luego se formuló el cuestionario no estructurado y posteriormente fue aplicado el 23 de marzo de 2017. Una vez fueron procesadas las respuestas se identificaron categorías para facilitar el análisis cualitativo e incluso posibilitar un análisis cuantitativo. La Tabla 2 presenta los objetivos, las preguntas y las respectivas categorías identificadas.

Vale la pena destacar que para la aplicación del instrumento para determinar la percepción, la pertinencia y las opiniones sobre la intervención del curso, fue definida una consigna general que guiara la respuesta de las preguntas formuladas. Dicha consigna fue la siguiente: “A continuación se presenta un breve cuestionario que busca determinar su percepción sobre la incorporación de actividades de lectura y escritura en el desarrollo del curso Desarrollo de Software II, por favor explique o argumente su respuesta”.

En relación con la primera pregunta aplicada, se identificó que los estudiantes reconocen que en otros cursos se aborda la escritura y la lectura, pero específicamente esta última, aunque de manera instrumental, es decir, la lectura no se usa para reflexionar sobre los temas que presenta, lo cual conduce a que no se generen espacios para el aprendizaje y mucho menos para la construcción de conocimiento a partir de la lectura. De hecho el $40 \%$ de las respuestas fueron asociadas a tal categoría; en consecuencia, podría decirse que no hay una relación importante entre dichos elementos en los contextos de las disciplinas a las que asisten los estudiantes, lo cual genera entonces una desventaja importante para el aprendizaje de los mismos, ya que como bien se menciona en [8] no basta con instrumentalizar la escritura y la lectura como prácticas académicas; es necesario comprender que dichos componentes son fundamentales para la construcción y apropiación de conocimiento. 
Tabla 2

Instrumento para valorar la percepción

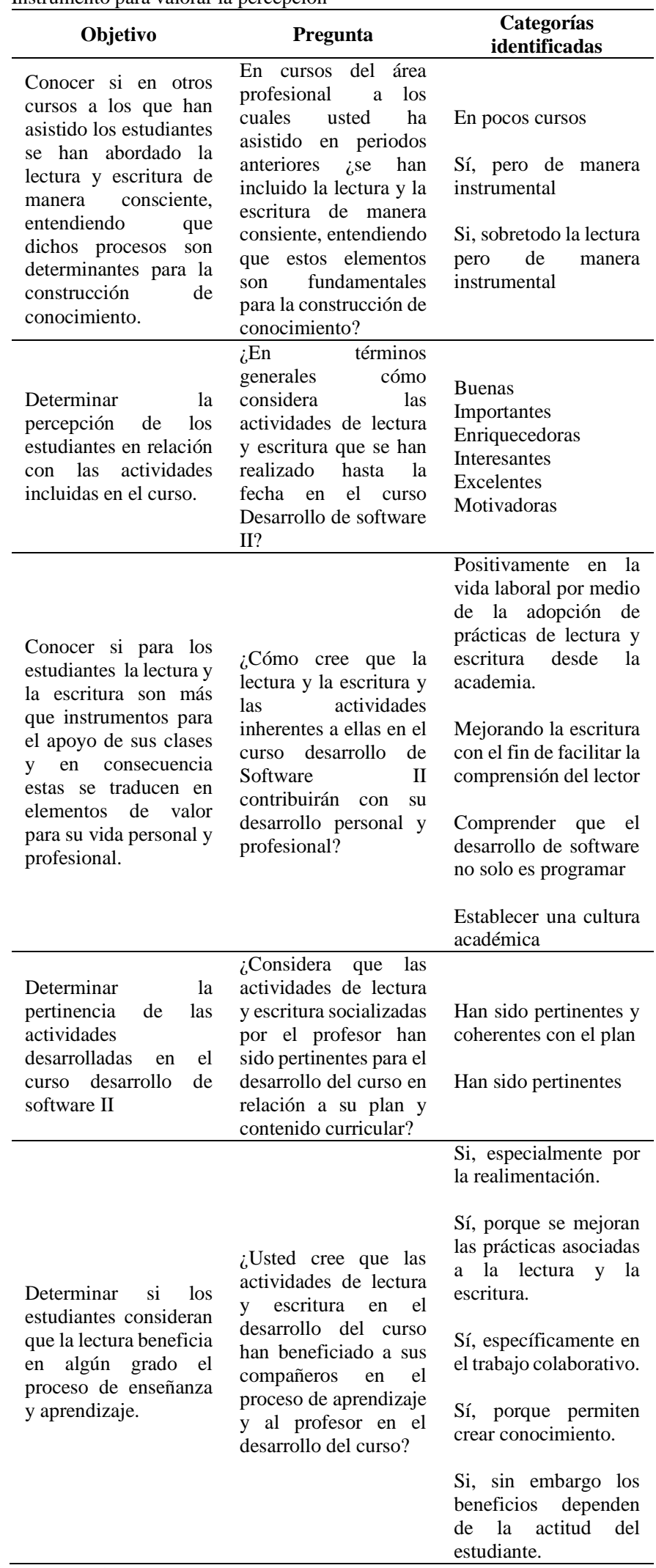

Fuente: Del autor

Adicionalmente es significativo exponer que para los estudiantes las actividades incluidas en las sesiones del curso significaron más que buenas experiencias, de hecho ellos comentaron que tales actividades resultaron incluso motivadoras, enriquecedoras e interesantes. Sin embargo se identificó que los efectos de las actividades incluídas en el curso dependen seriamente de la actitud de los estudiantes e incluso de su cultura académica, por tanto es necesario observar cada caso de manera particular con el fin de identificar problemas en el imaginario de los estudiantes sobre lo que realmente significa para ellos la escritura y la lectura, ya que es evidente que equivocadamente algunos de ellos entienden dichos componentes como apéndices o simples añadidos al proceso académico.

Por otra parte y en lo que respecta a la pregunta que buscó indagar sobre la concepción de la lectura y la escritura en los estudiantes, es importante comentar que la totalidad de ellos consideró que la lectura y la escritura no solo tienen un alcance en lo académico, de hecho manifestaron explícitamente la importancia de dichos componentes en la dinámica diaria de su vida personal y claramente en los efectos en el éxito laboral. En consecuencia, es posible afirmar que los estudiantes recomponen de alguna manera su imaginario de la escritura y la lectura de modo que comprenden su significado y la importancia de su apropiación como habilidades blandas, que son sin lugar a dudas significativas no solo para el éxito académico sino también para el éxito personal y laboral, en la medida que se traducen en aptitudes sociales que son reconocidas por empleadores en el evento que estudiantes o jóvenes recién graduados busquen empleos. Ejemplos de dichas habilidades pueden ser: Trabajo en equipo, capacidad comunicativa y flexibilidad.

En lo que respecta a la pertinencia de las actividades involucradas en el curso, se identificó que el 95\% de estudiantes considera que fueron apropiadas y coherentes con el plan presentado al inicio del periodo académico, lo anterior fue posible gracias a la adecuada contextualización de las actividades a la luz de la disciplina, por tanto, los artículos objeto de lectura modelada, corresponden a textos de ingeniería de software, modelos de calidad en software y especificación de requisitos. Adicionalmente las actividades de escritura orbitaron alrededor de la producción de textos que son habituales en la vida productiva de un ingeniero de software, en consecuencia se asignó la elaboración de procedimientos, manuales y guías relacionadas al proceso de desarrollo de software y al producto resultante de dicho proceso.

Con lo anterior, se encuentra que los estudiantes, específicamente el $50 \%$ de ellos valoraron de manera significativa el proceso de realimentación continua que se posibilitó durante las clases. De hecho comprenden que prácticas relacionadas con la escritura y la lectura son insumos fundamentales para el aprendizaje de manera individual y sobre todo de manera colectiva y colaborativa, y manifiestan que tales prácticas tienen un alcance que posibilita el mejor desempeño del profesor y contribuyen a la construcción de conocimiento y apropiación de conceptos propios de su disciplina.

Finalmente, es conveniente poner a consideración los siguientes cuestionamientos ¿Qué tan dispuesto debe estar un profesor para la incorporación de actividades de escritura y lectura en su disciplina y labor docente? ¿Cuál es el límite en relación con el plan o programa del curso que debe ser abordado 
con prácticas de dicha naturaleza? Lo que sí es claro es que el número de estudiantes matriculados es un factor determinante para la inclusión de actividades de este tipo; de igual manera, es necesario destacar que hay asignaturas o cursos en los que simplemente incorporar tales actividades resulta significativamente complejo. Para el caso específico del curso Desarrollo de Software II, de los programas de Ingeniería de Sistemas y Tecnología en Sistemas de Información de la Universidad del Valle, sede Tuluá, la incorporación de la lectura y la escritura resultó ser una actividad enriquecedora no solo para los estudiantes sino también para el profesor a cargo del curso. En consecuencia, es cuerdo pensar que en cursos de naturaleza similar, en cuanto a número de matriculados, intensidad horaria y objetivos del curso sea posible replicar ejercicios como los presentados en este artículo y por tanto realimentar sus resultados o contribuir con una discusión al respecto.

\section{Conclusiones}

La incorporación de rejillas resultó fundamental para el adecuado desarrollo del curso, de hecho usar dichas rejillas para la evaluación facilita esa labor al docente, porque proveen los elementos y criterios para proceder a la evaluación, generando en los estudiantes una visión completa de su nota cuantitativa, lo que implica que los estudiantes impugnen menos las calificaciones asignadas por el docente y asimilen los resultados de una manera constructiva y objetiva.

También, y específicamente en relación con los resultados del curso, se destaca que el 100\% de los 21 matriculados aprobó la asignatura, de hecho, solo el $28 \%$ de ellos obtuvieron notas entre 3 y 4. Esto se relaciona con la calidad de los documentos entregados por los estudiantes de forma grupal, es decir, se observó una evidente mejora en la especificación de requisitos, elaboración de manuales técnicos y construcción de documentos asociados a un proyecto de naturaleza software. En consecuencia, podría afirmarse que la incorporación de la lectura y la escritura, así como enfoques de trabajo colaborativo y ambientes constructivistas influyen de manera positiva en los resultados cuantitativos de los estudiantes y claramente en la valoración cualitativa de los mismos.

Por otro lado, la incorporación de la lectura y la escritura en la dinámica del curso no traumatizó el plan o programación del mismo, de hecho tales actividades estuvieron tan relacionadas con los contenidos del curso que este último se desarrolló de manera habitual en lo que tiene que ver con el plan o programa establecido para ello. También y en lo referente a la percepción de los estudiantes en relación con los temas incluidos en el curso, se encuentra que estos últimos gozan de aceptación y aprobación. Se evidencia que tal aceptación esta permeada por un nivel de consciencia tal, que conduce a concluir que los estudiantes pudieron comprender el valor epistémico de la lectura y la escritura, es decir, dichos componentes como generadores de conocimiento. Sin embargo y apelando a la prudencia, es imperativo mencionar que más allá de la nueva concepción de la lectura y la escritura que pueda habitar en los estudiantes, se destacan ellos como sujetos críticos que saben ahora que tanto la lectura como la escritura son procesos inacabados $\mathrm{y}$, por tanto, en constante refinamiento $\mathrm{y}$ apropiación.

\section{Referencias}

[1] Carlino, P., Alfabetización académica: Un cambio necesario, algunas alternativas Posibles. EDUCERE. [en línea]. 6(20), pp. 409-420, 2003. Disponible en: http://www.redalyc.org/pdf/356/35662008.pdf

[2] Garay, A., Integración de los jóvenes en el sistema universitario; prácticas sociales, académicas y de consumo cultural. [en línea]. Ediciones Pomares S.A. Barcelona, España. 2004. Disponible en: Http://Www.Scielo.Org.Mx/Pdf/Peredu/V27n107/N107a10.Pdf

[3] Universidad del Valle. Reporte socioeconómico estudiantil de la sede Tuluá, 2017.

[4] Natale, L. y Stagnaro, D., Desarrollo de habilidades de lectura y escritura en la trayectoria académica del ingeniero: La experiencia de un programa desafiante e innovador. Revista Argentina de Enseñanza de la Ingeniería. 2(3), pp. 45-52, 2013. ISBN 2250-6608

[5] Carrillo, L. y Orozco, O., La administración de conocimientos en las organizaciones que desarrollan sistemas de información: Análisis de la concepción de conocimiento. En: XVII Congreso Internacional de Contaduría, Administración e Informática. México, D.F., México. [en línea]. 2012. Disponible en: http://congreso.investiga.fca.unam. $\mathrm{mx} / \mathrm{docs} / \mathrm{xvii} / \mathrm{docs} / \mathrm{M} 03 . p d f$

[6] Torres, E., Aballay, L., Ferrarini, C., Zapata, S., Collazos, C., Guiraldo, F. y Ochoa, S., Enseñanza de ingeniería de software en un escenario distribuido y colaborativo. En: XI Workshop de Investigadores en Ciencias de la Computación. pp. 434-437, 2009. Disponible en: http://sedici.unlp.edu.ar/handle/10915/19789

[7] Ramírez, R. y López, G., La lectura y escritura en la formación de los ingenieros. En: Encuentro internacional de educación en ingeniería, ACOFI, [en línea]. 2014.2 Disponible en: https://www.researchgate.net/publication/277596711_la_lectura_y_la_escritu ra_en_la_formacion_de_los_ingenieros

[8] Carlino, P., El proceso de escritura académica: Cuatro dificultades de la enseñanza universitaria. EDUCERE. 8(26), pp. 321-327, 2004. ISSN 13164910.

[9] Elliot, J., La investigación-acción en la educación. Ediciones Morata. Cuarta Edición. [en línea]. 2000.2 Disponible en: http://www.terras.edu.ar/biblioteca/37/37ELLIOT-Jhon-Cap-1-y-5.pdf

[10] Latorre, A., Investigación-acción: Conocer y cambiar la práctica educativa. Editorial Grao, [en línea]. 2003. Disponible en: https://www.academia.edu/download/35282480/11_Latorre-Inv-Acc-cap1.pdf

[11] Castello, M., Lñesta, A., Miras, M., Solé, I., Teberosky, A. y Zanotto, M., Escribir y comunicarse en contextos científicos y académicos: Conocimientos y estrategias. Editorial Graó. 2007.

R.D. Estrada-Esponda, recibió el título de Tec. en Sistemas de Información e Ing. de Sistemas en la Universidad del Valle, en los años 2011 y 2014 respectivamente; actualmente adelanta la Maestría en Administración en la misma institución de educación superior, además se desempeña como profesor ocasional tiempo completo en la Universidad del Valle, sede Tuluá y es desarrollador de software con una experiencia laboral de más de 3 años en dicha actividad. Pertenece al programa interinstitucional para el fortalecimiento de la investigación y el posgrado del pacífico como investigador y además es instructor nacional seleccionado para los clubes de ciencia de Colombia 2017. Sus intereses en investigación incluyen la ingeniería de software, gestión del conocimiento, educación y user experience.

ORCID: 0000-0002-6849-1278 